\title{
Direct Instantons in QCD Nucleon Sum Rules
}

\author{
Hilmar Forkel and Manoj K. Banerjee \\ Department of Physics, University of Maryland, College Park, Maryland 20742-4111 (U.S.A.)
}

February 1993

hep-ph/9309232

\begin{abstract}
We study the role of direct (i.e. small-scale) instantons in QCD correlation functions for the nucleon. They generate sizeable, nonperturbative corrections to the conventional operator product expansion, which improve the quality of both QCD nucleon sum rules and cure the long-standing stability problem, in particular, of the chirally odd sum-rule.

12.38.Lg, 14.20.Dh
\end{abstract}

Typeset using REVTEX 
QCD sum rules, introduced by Shifman, Vainshtein and Zakharov (SVZ) [1], provide a systematic, nonperturbative framework for the calculation of hadron properties. They have been intensely studied and applied over the last decade and produced the most exhaustive model-independent analysis of hadron properties to date.

The sum-rule approach is based on the comparison of two "dual" descriptions for correlation functions of hadronic currents, in terms of quarks and gluons on the left-hand side (LHS) and in terms of hadrons on the right-hand side (RHS). The RHS uses a simple parametrization of the spectral function in terms of the hadron parameters, such as mass, overlap with the source current and continuum threshold. The QCD calculation on the LHS employs a non-perturbative operator-product expansion (OPE). Long-distance bulk properties of the physical vacuum are efficiently parametrized in terms of the vacuum expectation values of composite quark-gluon operators ("condensates"), which are independent of the hadron considered. The short-distance physics is contained in the perturbatively calculated Wilson coefficients. The inverse renormalization scale of the operators, $\mu^{-1}$, serves as the dividing line separating long and short distances.

Both sides of the sum rules are then Borel-transformed, and the hadron parameters are determined by fitting the two sides in the fiducial region, i.e. in the range of Borel mass values in which both descriptions of the correlator are expected to be adequate. The quality of this fit is the only intrinsic criterion for the accuracy and reliability of the sum rules. If it is met sufficiently well, the resulting hadron parameters will be approximately independent of the Borel mass in the fiducial region. The nucleon sum rules, however, do not show such a stability plateau, despite many improvements over the last decade [2]. It seems that some relevant physics in the fiducial region (around $1 \mathrm{GeV}$ ) is missing in the OPE. In this letter we suggest that small-size instantons [3], termed "direct" by SVZ, provide the dominant part of this physics.

Instantons [4] are classical solutions of the euclidean Yang-Mills equation. Due to the infrared complexities of QCD, their quantum properties and vacuum distribution cannot yet be derived from first principles. A consistent picture of their importance and bulk 
features has been established, however, by extensive phenomenological [5, [6], analytical [7] and numerical studies (in the instanton liquid model [6] and on the lattice, e.g. in ref. [8]). They indicate, in particular, that the average instanton size $\rho_{c}$ in the vacuum is considerably smaller than the average separation $R$ between instantons [6]:

$$
\rho_{c} \simeq \frac{1}{3} \mathrm{fm}, \quad R \simeq 1 \mathrm{fm}
$$

Most of the contributions of these rather small instantons to the correlation function are ignored in the conventional OPE: As the scale of these fields is smaller than the inverse renormalization scale (taken around $\mu \simeq 0.5 \mathrm{GeV}$ ), they would contribute to the Wilson coefficients, but do not show up in the perturbative evaluation.

The aim of our paper is to calculate the leading instanton contributions to the nucleon correlator, using an instanton size-distribution [6] in accordance with the bulk features (11), and to study their effects in the nucleon sum rules. We do not use the detailed assumptions of the above-mentioned model calculations.

The instanton contributions are mediated mainly by the quark zero-modes [9] in the instanton background field. Due to their particular chiral and color properties, the magnitude of instanton effects is channel dependent. Even if they can e.g. be safely neglected in the vector and axial-vector channel, they play a dominant role in the pseudoscalar sum rules [10] (and more generally in the spin-0 channel), where the conventional OPE was known to fail [1].

Our expectation of sizeable instanton effects in the nucleon sum rules is mainly based on parallels with the pseudoscalar sector. The interpolating fields (see below) in the nucleon correlator contain spin-0 diquarks, which receive zero-mode contributions of the same order as the pseudoscalars. Furthermore, the magnitude of the nucleon correlator at distances around 1 - $2 \mathrm{fm}$ is much larger than the perturbative contribution [11], which is reminiscent of the strongly attractive correlations due to instantons in the pseudoscalar channel.

Recently, Dorokhov and Kochelev [12] made a first attempt to calculate instanton contributions to the nucelon sum rule. However, as we will outline in the course of this paper, 
we do not agree with their results and many of their conclusions.

The QCD nucleon sum-rules are based on the correlation function

$$
\mathrm{i} \int d^{4} x \mathrm{e}^{\mathrm{i} q x}<0|T \eta(x) \bar{\eta}(0)| 0>=\not \Pi_{q}\left(q^{2}\right)+\Pi_{m}\left(q^{2}\right)
$$

evaluated at intermediate space-like momentum transfer, $Q^{2} \equiv-q^{2} \simeq 1 \mathrm{GeV}$. We will use the most general interpolating field of the nucleon (with minimal mass dimension):

$$
\eta(x)=\epsilon_{a b c}\left\{\left[u_{a}^{T}(x) C d_{b}(x)\right] \gamma_{5} u_{c}(x)+t\left[u_{a}^{T}(x) C \gamma_{5} d_{b}(x)\right] u_{c}(x)\right\}
$$

Here, $u, d$ denote the up and down quarks, $C$ is the charge-conjugation Dirac matrix, and the real coefficient $t$ specifies the linear combination of the currents containing scalar and pseudoscalar diquarks. Ioffe's current [2] corresponds to $t=-1$.

We intend to evaluate the dominant instanton contributions to the correlator eq. (2) at distances relevant for the sum rules, $q^{-1} \simeq 0.2 \mathrm{fm}[\mathbb{1}]$. As these distances are small compared to the average separation $R \simeq 1 \mathrm{fm}$ between instantons, contributions from a single instanton should dominate multi-instanton effects. The small average size of the instantons further implies a sufficiently small gauge coupling $g\left(\Lambda \rho_{c}\right)$, which allows us to calculate the correlator in semiclassical approximation.

To this end, we evaluate eq. (2) with the help of the quark propagator in an instanton background field [5], which is dominated by the zero-mode contribution

$$
S_{0}^{ \pm}(x, y)=\frac{\psi_{0}^{ \pm}(x) \psi_{0}^{ \pm \dagger}(y)}{m^{*}(\rho)}+O\left(\left(\rho m^{*}\right)^{-1}\right)
$$

The superscript \pm refers to an instanton/anti-instanton of fixed size $\rho$ and position $x_{0}$. The zero-mode solutions $\psi_{0}^{ \pm}$have been derived by 't Hooft in ref. [9]. Due to interactions with QCD vacuum fields, the quarks acquire an effective mass, $m^{*}(\rho)=m_{q}-\frac{2}{3} \pi^{2} \rho^{2}<0|\bar{q} q| 0>$, which has been given by SVZ [13]. (In the following, we will neglect the small current quark masses $m_{q}$.)

Due to the symmetry properties of the zero-mode solution, maximally two of the three quarks created by the interpolating field propagate in zero-mode states. We approximate the 
propagation of the third quark in the continuum states [0] by the standard quark propagator used in the OPE and obtain

$$
\begin{aligned}
<0|T \eta(x) \bar{\eta}(0)| 0> & =\frac{1}{\pi^{4}}\left(\frac{c_{1}}{\pi^{2}} \frac{\not x}{x^{4}}+\frac{2 i c_{2}}{N_{c}}<0|\bar{q} q| 0>\right) \\
& \times \int d \rho n(\rho) \frac{\rho^{4}}{m^{* 2}(\rho)} \int d^{4} x_{0} \frac{1}{\left(\left(x-x_{0}\right)^{2}+\rho^{2}\right)^{3}} \frac{1}{\left(x_{0}^{2}+\rho^{2}\right)^{3}}
\end{aligned}
$$

where we define

$$
c_{1}=6\left(t^{2}-1\right), \quad c_{2}=\frac{1}{8}\left[13\left(t^{2}+1\right)+10 t\right] .
$$

Due to translational and gauge invariance, the integration over all collective coordinates of the instanton except the scale $\rho$ is straightforward. The broken scale invariance, however, gives rise to a $\rho$-dependent weighting factor, the instanton size-density $n(\rho)$. As motivated above, we will use the simple form proposed in ref. [5],

$$
n(\rho)=n_{c} \delta\left(\rho-\rho_{c}\right),
$$

which incorporates the already described features and scales of the instanton distribution and the quite sharply peaked (half-width $\simeq 0.1 \mathrm{fm}$ ), almost gaussian result of Monte-Carlo simulations [14.

The Fourier transform of (5) contains the instanton contribution to the invariant amplitudes $\Pi_{q}\left(q^{2}\right)$ and $\Pi_{m}\left(q^{2}\right)$ in (2). Their subsequent Borel transform, required in the sum rules, leads to quite complicated integrals [15] which we evaluate numerically, but do not write down explicitly here. Instead we present a saddle-point approximation, which is more transparent and allows for a direct comparison with the work of [12]:

$\hat{\Pi}_{q}\left(M_{B}^{2}\right)=\frac{c_{1}}{64 \sqrt{\pi} \pi} \frac{n_{c}}{m^{* 2} \rho_{c}^{2}}\left[\frac{64}{10 \sqrt{\pi}}\left(\frac{1}{z^{2}}-\frac{24}{7} \frac{1}{z^{4}}\right)+\left(z^{2}+4+9 \frac{1}{z^{2}}\right) \frac{e^{-z^{2}}}{z}+3 \sqrt{\pi}\left(\frac{1}{z^{2}}+\frac{15}{2 z^{4}}\right) \operatorname{erfc}(z)\right]$

and

$$
\hat{\Pi}_{m}\left(M_{B}^{2}\right)=\frac{-\sqrt{\pi} c_{2}}{4 N_{c}} \frac{<\bar{q} q>n_{c}}{m^{* 2}}\left(z^{3}-\frac{3}{2} z\right) e^{-z^{2}}
$$


where $M_{B}$ is the Borel mass, $\operatorname{erfc}(z)$ is the complementary error function [16] and we define $z=M_{B} \rho_{c}$. Note that the instanton contribution to $\hat{\Pi}_{q}$ vanishes for the Ioffe current (i.e. $\left.c_{1}=0\right)$.

We eliminate the $n_{c}$ dependence of (8) and (9) with the self-consistency relation [17]:

$$
<\bar{q} q>=-2 \int d \rho \frac{n(\rho)}{m^{*}(\rho)}=-2 \frac{n_{c}}{m^{*}\left(\rho_{c}\right)} .
$$

While the assumption of a completely zero-mode-induced quark condensate is rather strong, our final results will not depend on it, because the numerical value obtained from (10) is almost exactly equal to the phenomenologically estimated value.

Before combining eqs. (8) and (9) with the standard OPE terms of the nucleon sum rules [2], we have to address the issue of double counting instanton physics. The conventional OPE already accounts for all nonpertubative vacuum fields (including instantons) with scales below the renormalization scale $\mu$ in the condensates, whereas the whole $M$-dependence, logarithms and powers of $M^{-2}$, comes from the perturbatively calculated Wilson coefficients.

The $M$-dependence of the instanton contributions, however, is exponential in all terms of $\hat{\Pi}_{m}$ and in all but two terms of $\hat{\Pi}_{q}$. Hence these terms constitute new, nonpertubative contributions to the Wilson coefficients, originating from small-instanton physics. $\hat{\Pi}_{q}$, however, contains also two power terms [18], which represent instanton contributions to the condensates of dimension 6 (four-quark condensates) and 8 [19]. In this letter we will restrict the conventional OPE to operators of dimension $\leq 6$, so that double counting has to be prevented only for the four-quark condensates.

The standard sum rule practice is to approximate the four-quark condensates by factorizing them into two-quark condensates, and it is known that this approximation doesn't always work well for the instanton contribution. Indeed, the four-quark condensate term in eq. (8) is considerably larger than the corresponding factorized OPE condensate, which we will therefore omit completely.

The LHS of the sum rules can now be obtained by adding the invariant amplitudes, (8) and (9), to their standard OPE counterpart [2] with the corrected four-quark condensate. 
On the RHS, these amplitudes are written in a (Borel-transformed) Lehmann representation with the usual pole-continuum approximation for the spectral functions [2]. Equating both sides and transferring the continuum contribution to the left, we obtain the two sum rules

$$
\begin{array}{r}
2 c_{3} M^{6} A_{2}(M, W)+2 c_{3} \pi^{2} M^{2}<\frac{\alpha}{\pi} G_{\mu \nu} G^{\mu \nu}>A_{0}(M, W)+\frac{1}{3} c_{5}(4 \pi)^{4}<\bar{q} q>^{2}+\frac{96}{5} c_{1} \rho_{c}^{-6}\left(1-\frac{24}{7} z^{-2}\right) \\
+3 c_{1} \sqrt{\pi} \rho_{c}^{-6}\left[z^{3}+4 z+\frac{9}{z}+3 \sqrt{\pi}\left(1+\frac{15}{2} z^{-2}\right) e^{z^{2}} \operatorname{erfc}(z)\right] e^{-z^{2}}=\tilde{\lambda}_{N}^{2} e^{-m^{2} / M^{2}}
\end{array}
$$

and

$$
-(4 \pi)^{2}<\bar{q} q>\left[c_{4} M^{4} A_{1}(M, W)-\frac{c_{1}}{8} m_{0}^{2} M^{2} A_{0}(M, W)+c_{2} \sqrt{\pi} \rho_{c}^{-4} z^{3}\left(z^{2}-\frac{3}{2}\right) e^{-z^{2}}\right]=m \tilde{\lambda}_{N}^{2} e^{-m^{2} / M^{2}}
$$

where the

$$
A_{n}(M, W)=1-e^{-W^{2} / M^{2}}\left[1+\sum_{m=1}^{n} \frac{1}{m}\left(\frac{W^{2}}{M^{2}}\right)^{m}\right]
$$

contain the continuum contributions and

$$
c_{3}=\frac{1}{8}\left[5\left(t^{2}+1\right)+2 t\right], \quad c_{4}=\frac{1}{4}\left[7 t^{2}-2 t-5\right], \quad c_{5}=\frac{1}{8}(t-1)^{2} .
$$

Let us now compare our results with those of ref. [12], where semi-classical instanton contributions to the nucleon sum rules are also evaluated. We find four additional terms in $\hat{\Pi}_{q}$ and one in $\hat{\Pi}_{m}$, all with exponential Borel-mass dependence. These terms, which apparently have been missed in [12], are in no way subleading and their omission has drastic consequences: 1.) The spectral function of $\hat{\Pi}_{q}$ becomes negative in the whole fiducial domain, thus violating its very general positivity bound [20]. 2.) For the same reason, the (usually more reliable) $\hat{\Pi}_{q}$ sum rule becomes unstable and has to be abandoned. Half of the physical information and the valuable consistency check between the two sum rules is lost. 3.) A numerically important part of $\hat{\Pi}_{m}$ is missing and the relation between nucleon mass and quark condensate, usually manifest in the "Ioffe formula" [2], is obscured.

Furthermore, the authors of [12] used the (only qualitatively reliable) saddle-point approximation to evaluate the sum rules, which induces substantial errors $(\sim 30 \%)$ in the 
nucleon parameters. (As already mentioned, our results are based on the exact, numerical evaluation of the instanton contribution.) Finally, double-counting of the four-quark condensate is not addressed in [12] and the 4- and 5-dimensional condensates are neglected.

The free parameters to be determined in the sum rules are the continuum threshold $W$, the nucleon mass $m$ and $\lambda_{N}$, the overlap of the wave packet created by $\eta$ with the nucleon $\left(<0|\eta(0)| N_{\alpha}(k)>=\lambda_{N} u_{\alpha}(k), \tilde{\lambda}_{N} \equiv(4 \pi)^{2} \lambda_{N}\right)$. We obtain their values by minimizing the difference, defined by the measure $\delta$ of ref. [2], between the two sides of the sum rules (11) and (12) in the fiducial Borel mass domain $0.8 \mathrm{GeV} \leq M \leq 1.2 \mathrm{GeV}$. In this letter we choose $t=-1.1$ [21], corresponding to the optimal current of Espriu et al. [2] and close to the Ioffe current. For any choice of the current, however, the instanton contribution will be substantial in at least one sum rule.

Figure 1a shows the OPE contribution, the instanton contribution and their sum, the complete LHS, in comparison with the pole term $\tilde{\lambda}_{N}^{2} e^{-m^{2} / M^{2}}$ for the $\not 1$ sum rule (11). Figure 1b shows the same curves, divided by the nucleon mass $m$, for the sum rule (12). As expected, the instanton contributions to the $\not$ sum-rule are rather small for our current, but still improve the fit quality $(\delta=1.2 \%)$. The nucleon parameters become $m=0.88 \mathrm{GeV}$, $W=1.43 \mathrm{GeV}$ and $\tilde{\lambda}_{N}^{2}=2.90 \mathrm{GeV}^{6}$.

In the traditionally less stable $m$ sum-rule, however, the instanton contributions become comparable to the OPE contributions and improve the fit quality dramatically. Their $M$ behaviour balances the OPE terms over the full Borel-mass interval to bring their sum very close $(\delta=0.03 \%)$ to the pole contribution. The nucleon parameters become $m=0.90 \mathrm{GeV}$, $W=1.60 \mathrm{GeV}$ and $\tilde{\lambda}_{N}^{2}=5.68 \mathrm{GeV}^{6}$.

The different magnitude of the instanton contributions in the two sum rules implies that a simultaneous fit of both sum rules will not yield their individually optimal parameter values. Indeed, the nucleon mass increases $(m=1.06 \mathrm{GeV})$ and the fit quality does not improve as significantly as in the $m$ sum-rule. This might indicate some missing physics in the $\not$ sum-rule. Radiative $\mathrm{O}\left(\alpha_{s}\right)$ corrections to the conventional OPE, e.g., are known to contribute at the same order as the leading perturbative term to the $\not$ sum-rule and their 
inclusion lowers the nucleon mass [22].

As mentioned above, the quality of the sum rules is reflected in the approximate Borelmass independence of $m(M)$ and $\tilde{\lambda}_{N}^{2}(M)$, obtained by solving the optimized sum rules. The instanton-improved sum rules leave both quantities quite insensitive to the Borel mass (Fig. 3). The usually less stable $m$ sum-rule, which receives the main instanton corrections, shows a particularly pronounced improvement and generates perfect stability plateaus over the whole fiducial region.

The extension of our investigation to other baryons and to finite density and temperature is in progress. This work was supported in part by the US Department of Energy under Grant No. DE-FG02-93ER-40762. 


\section{REFERENCES}

[1] M.A. Shifman, A.I. Vainshtein and V.I. Zakharov, Nucl. Phys. B 147, 385, 448 (1979)

[2] B.L. Ioffe, Nucl. Phys. B 188, 317 (1981), Nucl. Phys. B 191, 591 (1981); V.M. Belyaev and B.L. Ioffe, Sov. Phys. JETP 56, 493 (1982); Y. Chung et al., Nucl. Phys. B 197, 55 (1982); Espriu et al., Nucl. Phys. B 214, 285 (1983); D. B. Leinweber, Ann. Phys 198, $203(1990)$

[3] For simplicity we will often use the term "instanton" generically, i.e. for instanton and/or anti-instanton.

[4] A.A. Belavin et al., Phys. Lett B 59, 85 (1975)

[5] E.V. Shuryak, Nucl. Phys. B 203, 93,116 (1982)

[6] E.V. Shuryak, The QCD Vacuum, Hadrons and the Superdense Matter (World Scientific Pub. Co., Singapore, 1988) and references therein

[7] D.I. Diakonov and V.Yu. Petrov, Nucl. Phys. B 245, 259 (1984); Phys. Lett. B 147, 351 (1984); Nucl. Phys. B 272, 457 (1986)

[8] M.-C. Chu and S. Huang, Phys. Rev. D 45, 2446 (1992)

[9] G. 't Hooft, Phys. Rev. Lett. 37, 8 (1976); Phys. Rev. D 14, 3432 (1976)

[10] E.V. Shuryak, Nucl. Phys. B 214, 237 (1983)

[11] E.V. Shuryak, Rev. Mod. Phys. 65, 1 (1993)

[12] A.E. Dorokhov and N.I. Kochelev, Z.Phys.C 46, 281 (1990)

[13] M.A. Shifman, A.I. Vainshtein and V.I. Zakharov, Nucl. Phys. B 163, 46 (1980)

[14] E.V. Shuryak and J.J.M. Verbaarschot, Nucl. Phys. B 341, 1 (1990)

[15] H. Forkel and M.K. Banerjee, in preparation 
[16] M. Abramowitz and I.A. Stegun, Handbook of Mathematical Functions, (National Bureau of Standards, Washington D.C., 1972)

[17] C.G. Callen Jr., R. Dashen and D.J. Gross, Phys. Rev. D 17, 2717 (1978), D.G. Caldi, Phys. Rev. Lett. 39, 121 (1977)

[18] They are consistent with the results of [23], which restrict instanton-generated power corrections in hadron correlators to only a few low-dimensional terms (in the absence of radiative corrections).

[19] The distinction between exponential and power behaviour remains valid beyond the saddle-point approximation. The instanton-generated power terms in eq. (\$), in particular, are calculated exactly.

[20] J.D. Bjorken and S.D. Drell, Relativistic Quantum Fields (McGraw-Hill, New York, 1965)

[21] We have evaluated the sum rules in a large range of values for t. Their current dependence, both in fit quality and results, is reduced by the instanton contributions, as compared to the standard OPE. Due to space limitations, a detailed discussion will be postponed to a forthcoming publication [15].

[22] A.A. Ovchinnikov et al., Sov.J.Nucl.Phys.48, 358 (1988)

[23] M.S. Dubovikov and A.V. Smilga, Nucl. Phys. B 185 (1981) 109; 


\section{FIGURES}

FIG. 1. a) The OPE (dashed line) and instanton (dot-dashed line) contributions to the $\not$ sum rule. Their sum (dotted line) is compared to the pole contributions (solid line). b) The same for the $m$ sum rule. The sum of OPE and instanton contributions is practically indistinguishable from the pole term.

FIG. 2. The nucleon mass as a function of the Borel mass from (a) the $\not$ sum rule, (b) the $m$ sum-rule and (c) the combined fit. The coupling $\tilde{\lambda}_{N}^{2}$ from (d) the $\not d$ sum-rule and (e) the $m$ sum-rule. 